PROCEEDINGS OF THE

AMERICAN MATHEMATICAL SOCIETY

Volume 139, Number 9, September 2011, Pages 3083-3097

S 0002-9939(2011)10732-9

Article electronically published on January 24, 2011

\title{
MORE ABOUT DICRITICALS
}

\author{
SHREERAM S. ABHYANKAR
}

(Communicated by Bernd Ulrich)

\begin{abstract}
This paper attempts to characterize the dicritical set of a rational function which generates a special pencil at a simple point of an algebraic or arithmetical surface.
\end{abstract}

\section{INTRODUCTION}

The study of dicritical divisors started in Section 5 of $\mathrm{Ab5}$ ] was continued in $\mathrm{AbH}$ and $\mathrm{AbL}$. The main results of these two papers will be restated as Propositions 2.1 and 2.2 of Section 2. In Section 3, we shall partly answer the following question (1.1) concerning dicritical divisors which was raised and labelled (6.4) in Section 6 of [Ab6]. In Section 2 we shall recall the notation used in the study of dicriticals including what is needed in (1.1).

Question 1.1. Given any finite $U \subset D(R)^{\Delta}=$ the set of all prime divisors in a two dimensional regular local domain $R$, consider the set $\mathfrak{D}^{*}(R, U)^{\dagger}$ of all nonzero elements $z$ in the quotient field $L$ of $R$ such that $z$ generates a special pencil at $R$ and the dicritical set $\mathfrak{D}(R, z)$ of $z$ in $R$ coincides with $U$. As a "sort of converse" of (2.2) we may ask, is it always true that $\mathfrak{D}^{*}(R, U)^{\dagger} \neq \emptyset$ ? If not true in general, for what $U$ is this true? In particular, given any $V \in D(R)^{\Delta}$ is it true for $U=\{V\}$ ? If not, then for which $V$ is it true?

Part of the required notation was introduced in $\mathrm{Ab}$, where a small beginning at answering the above question was made, and "more about dicriticals at a later opportunity" was announced. This is that opportunity. Both the papers are based on intense discussions with Bill Heinzer.

Note that $z$ generates a special pencil at $R$ means that $z=a / b$ where $a \neq 0 \neq b$ in $R$ with $b=x^{m}$ for some nonnegative integer $m$ and element $x$ in the maximal ideal $M(R)$ of $R$ such that $x \notin M(R)^{2}$. Hence the above question can be paraphrased in the equivalent language of complete ideals thus. Characterize those finite sets of distinct simple complete $M(R)$-primary ideals $J_{1}, \ldots, J_{h}$ for which there exist positive integers $u_{1}, \ldots, u_{h}$ such that the ideal $I=J_{1}^{u_{1}} \ldots J_{h}^{u_{h}}$ has a two-generated reduction $J=(a, b)$ with $a, b$ as above. Note that $J$ is a reduction of $I$ means $J \subset I$ and $J I^{n}=I^{n+1}$ for some nonnegative integer $n$.

Received by the editors January 30, 2010 and, in revised form, August 7, 2010

2010 Mathematics Subject Classification. Primary 14A05.

Key words and phrases. Criticals, special pencil.

(C)2011 American Mathematical Society Reverts to public domain 28 years from publication 


\section{NotATiOn AND TERMinOLOGY}

In addition to the notation and terminology introduced in Section 2 and Remark (4.0) of [Ab7, we shall use the following notation and terminology.

Recall that $L^{\times}=$the set of all nonzero elements of $L$, and $\mathfrak{D}(R, z)$ (resp: $\left.\mathfrak{D}(R, z)^{\sharp}, \mathfrak{D}(R, z)^{b}\right)$ is the set of all dicritical divisors (resp: sharp dicritical divisors, flat dicritical divisors) of $z \in L^{\times}$in $R$, i.e., the set of all $V \in D(R)^{\Delta}$ such that $z$ is residually transcendental (resp: a generator, a polynomial) over $R$ relative to $V$.

We introduce the inverses of the $\mathfrak{D}$ functions by saying that for any finite subset $U$ of $D(R)^{\Delta}$ we let $\mathfrak{D}^{*}(R, U)$ (resp: $\left.\mathfrak{D}^{*}(R, U)^{\sharp}, \mathfrak{D}^{*}(R, U)^{b}\right)$ be the set of all $z \in L^{\times}$ such that $\mathfrak{D}(R, z)$ (resp: $\left.\mathfrak{D}(R, z)^{\sharp}, \mathfrak{D}(R, z)^{b}\right)$ equals $U$. We also let $\mathfrak{D}^{*}(R, U)^{\dagger}=$ the set of all $z \in L^{\times}$such that $z$ generates a special pencil at $R$ and $\mathfrak{D}(R, z)=U$.

Concerning dicritical divisors, Proposition 2.1 is proved in my joint paper $\mathrm{AbH}$ ] with Heinzer, and Proposition 2.2 is proved in my joint paper $\mathrm{AbL}$ with Luengo.

Proposition 2.1. Let $R$ be a two dimensional regular local domain with quotient field $L$. Let $U$ be any finite set of prime divisors of $R$. Then there exists $z \in L^{\times}$ such that $\mathfrak{D}(R, z)=U$. Moreover, if the field $R / M(R)$ is infinite, then there exists $z \in L^{\times}$such that $\mathfrak{D}(R, z)^{\sharp}=\mathfrak{D}(R, z)^{b}=\mathfrak{D}(R, z)=U$.

Proposition 2.2. Let $R$ be a two dimensional regular local domain with quotient field L. Let $z \in L^{\times}$be such that $z$ generates a special pencil at $R$. Then $z$ generates a polynomial pencil in $R$.

The proof of (2.1) is based on the theory of complete ideals developed by Zariski in Appendices IV and V of [ZaS], which we shall summarize in Propositions 2.3 and 2.4. Before stating these propositions, we shall introduce some relevant definitions.

An ideal $J$ in a ring $A$ is simple means (1) $J \neq A$ and (2) $J_{1}, J_{2}$ ideals in $A$ with $J=J_{1} J_{2} \Rightarrow J_{1}=A$ or $J_{2}=A$. If $A$ is a subring of a field $L$, then $\bar{D}(L / A)$ denotes the set of all valuation rings $V$ with quotient field $L$ such that $A \subset V$. If $A$ is a domain with quotient field $L$, then $J$ is a valuation ideal means it is the intersection of $A$ with an ideal in a ring which is a member of $\bar{D}(L / A)$. If $A$ is a normal domain with quotient field $L$, i.e., if $A$ is a domain which is integrally closed in its quotient field $L$, then $J$ is complete means it is an intersection of valuation ideals, and $J$ is normal means $J^{c}$ is complete for all $c \in \mathbb{N}_{+}=$the set of all positive integers. If $A$ is a normal domain, then by $\bar{C}(A)$ we denote the set of all nonzero complete ideals in $A$, and by the completion of an ideal $J$ in $A$ we mean the complete ideal $\bar{J}$ in $A$ obtained by putting $\bar{J}=\bigcap_{V \in \bar{D}(L / A)}((J V) \cap A)$; if $A$ is also local (= Noetherian quasilocal), then by $C(A)$ we denote the set of all $M(A)$-primary simple complete ideals in $A$.

Let $R \subset S$ be nonnull rings and let $J$ be an ideal in $R$. An element $x$ of $S$ is integral over $J$ means $f(x)=0$ for a univariate polynomial $f(Z)$ of the form

$\left.{ }^{*}\right) \quad f(Z)=Z^{n}+y_{1} Z^{n-1}+\cdots+y_{n}$ with $n \in \mathbb{N}_{+}$and $y_{i} \in J^{i}$ for $1 \leq i \leq n$.

A subset $T$ of $S$ is integral over $J$ means every $x \in T$ is integral over $J$. We may write $x / J$ (is) integral or $T / J$ (is) integral to indicate that $x$ is integral over $J$ or $T$ is integral over $J$ respectively. By the integral closure of $J$ in $S$ we mean the set of all elements of $S$ which are integral over $J$; note that the integral closure of $R$ in $S$ is a subring $\bar{R}$ of $S$, and the integral closure of $J$ in $S$ is an ideal in $\bar{R}$. Let 
$I$ be an ideal in $R$. We say that $J$ is a reduction of $I$ to mean that

$$
J \subset I \text { and } J I^{n}=I^{n+1} \text { for some } n \in \mathbb{N} \text {. }
$$

We may write $J / I$ (is a) reduction to indicate that $J$ is a reduction of $I$. By the Rees ring of $I$ relative to $R$ with variable $Z$ we mean the $\operatorname{ring} E_{R}(I)$ obtained by putting $E_{R}(I)=R[I Z]$. Note that $R[Z]$ is the univariate polynomial ring as a naturally graded homogeneous ring with $R[Z]_{n}=$ the set of all homogeneous polynomials of degree $n$ including the zero polynomial, and $n$ varying over $\mathbb{N}$. Now $E_{R}(I)$ is a graded subring of $R[Z]$. We make the convention that the reference to $R$ and $Z$ may be omitted when it is clear from the context. Thus we write $E(I)$ instead of $E_{R}(I)$. Assuming $I \subset M=$ a nonunit ideal in $R$, we define the form ring $F_{(R, M)}(I)$ of $I$ relative to $R$ with variable $Z$ by putting $F_{(R, M)}(I)=$ $E_{R}(I) / M E_{R}(I)$. Again we make the convention that the reference to $(R, M)$ and $Z$ may be omitted when it is clear from the context. Thus we write $F(I)$ instead of $F_{(R, M)}(I)$. Now $M E(I)$ is a homogeneous ideal in $E(I)$ and hence $F(I)$ becomes a graded ring. Note that, upon letting $F_{(R, M)}(I)_{n}$ or $F(I)_{n}$ be the $n$-th homogeneous component of $F(I)$ for all $n \in \mathbb{N}$ and upon letting $K=F(I)_{0}$, we have $F(I)=$ $K\left[F(I)_{1}\right]$ and, via the ring epimorphism $R \rightarrow K=R / M$, the $K$-module $F(I)$ becomes an $R$-module so that, for every $n \in \mathbb{N}$, the $K$-submodule $F(I)_{n}$ of $F(I)$ becomes an $R$-submodule of $F(I)$ and there is a canonical $R$-epimorphism $\mu_{n}$ : $I^{n} \rightarrow F(I)_{n}$ with kernel $M I^{n}$ such that

$$
\mu_{u+v}(y z)=\mu_{u}(y) \mu_{v}(z) \text { for all }(u, v, y, z) \in \mathbb{N} \times \mathbb{N} \times I^{u} \times I^{v} .
$$

Observe that $F(I)$ is isomorphic as a graded ring to the associated graded ring $\operatorname{grad}(R, I, M)$ of Definition (D3) on page 586 of [Ab4]. If $J \subset I$, then $\mu_{1}(J)$ is a $K$-submodule of $F(I)_{1}$ and $K\left[\mu_{1}(J)\right]$ is a homogeneous subring of $F(I)$; we denote this subring by $F_{(R, M)}(I, J)$ and we note that for its $n$-th homogeneous component, $F_{(R, M)}(I, J)_{n}=\mu_{n}\left(J^{n}\right)$ for all $n \in \mathbb{N}$. We call $F_{(R, M)}(I, J)$ the form ring of $(I, J)$ relative to $(R, M)$ with variable $Z$. Again we make the convention that the reference to $(R, M)$ and $Z$ may be omitted when it is clear from the context. Thus we write $F(I, J)$ and $F(I, J)_{n}$ instead of $F_{(R, M)}(I, J)$ and $F_{(R, M)}(I, J)_{n}$ respectively. Note that if $R$ is Noetherian and $M$ is a maximal ideal in $R$, then, for every $n \in \mathbb{N}$, $F(I)_{n}$ is a finite dimensional vector space over the field $R / M$ and $F(I)_{1} F(I)$ is the unique homogeneous maximal ideal in $F(I)$.

Reverting to a two dimensional regular local domain $R$ with quotient field $L$, given any $z \in L^{\times}$, we recall that the numerator ideal $a_{R}(z)$ of $z$ in $R$, the denominator ideal $b_{R}(z)$ of $z$ in $R$, and the first associated ideal $J_{R}(z)$ of $z$ in $R$ are defined by writing $z=a / b$ such that $a \neq 0 \neq b$ in $R$ have no nonunit common factor in $R$ and letting $a_{R}(z)=a R, b_{R}(z)=b R$, and $J_{R}(z)=(a, b) R$. We define the second associated ideal $I_{R}(z)$ of $z$ in $R$ by putting $I_{R}(z)=$ the integral closure of $J_{R}(z)$ in $R$. Recall that

$$
C(R)=\text { the set of all } M(R) \text {-primary simple complete ideals in } R \text {. }
$$

We are now ready to state the Zariski Propositions 2.3 and 2.4.

Proposition 2.3. In a normal domain, the completion of an ideal coincides with its integral closure.

Proposition 2.4. In a two dimensional regular local domain $R$ we have the following. 
(I) Given any $V \in D(R)^{\Delta}$ there is at least one and at most a finite number of $V$-ideals in $R$ which are members of $C(R)$. Labelling these members of $C(R)$ as

$$
M(R)=J_{0} \supsetneqq J_{1} \supsetneqq \cdots \supsetneqq J_{\nu},
$$

we get a bijection $\zeta_{R}: D(R)^{\Delta} \rightarrow C(R)$ by taking $\zeta_{R}(V)=J_{\nu}$. We call $\zeta_{R}$ the Zariski map of $R$. Also we call $\left(J_{i}\right)_{0<i \leq \nu}$ the simple $V$-ideal sequence of $R$.

(II) To describe the inverse map $\zeta_{R}^{-1}$ more explicitly we proceed thus. Given any $I \in C(R)$ let $\left(R_{0}, I_{0}\right)=(R, I)$. It can be shown that if $I_{0} \neq M\left(R_{0}\right)$, then there is a unique $R_{1} \in Q_{1}\left(R_{0}\right)$ such that upon letting $I_{1}=\left(R_{0}, R_{1}\right)\left(I_{0}\right)$ we have $I_{1} \neq R_{1}$. It can also be shown that $I_{1} \in C\left(R_{1}\right)$. If $I_{1} \neq M\left(R_{1}\right)$, then let $\left(R_{2}, I_{2}\right)$ be the pair such that $R_{2} \in Q_{1}\left(R_{1}\right)$ and $I_{2}=\left(R_{1}, R_{2}\right)\left(I_{1}\right) \neq R_{2}$, and so on. It can be shown that this process is finite. Thus we get a unique sequence $\left(R_{j}, I_{j}\right)_{0 \leq j \leq \nu}$ with $\nu \in \mathbb{N}$ such that $R_{j+1} \in Q_{1}\left(R_{j}\right)$ with $I_{j} \in C\left(R_{j}\right) \backslash\left\{M\left(R_{j}\right)\right\}$ and $I_{j+1}=\left(R_{j}, R_{j+1}\right)\left(I_{j}\right)$ for $0 \leq j<\nu$, and $I_{\nu}=M\left(R_{\nu}\right)$. We call this sequence the transform sequence of $(R, I)$. Let $V=o\left(R_{\nu}\right)$. Now clearly $\left(R_{j}\right)_{0 \leq j \leq \nu}$ is the finite QDT sequence of $R$ along $V$. It can be shown that the map $\eta_{R}: C(R) \rightarrow D(R)^{\Delta}$ given by $I \mapsto V$ is a bijection. Indeed $\eta_{R}=\zeta_{R}^{-1}$. Moreover, if the $V$ here is the same as the $V$ above, then the two values of $\nu$ coincide and we have $J_{i}=R \cap I_{\nu-i}$ for $0 \leq i \leq \nu$. We call $\eta_{R}$ the inverse Zariski map of $R$. Note that $\zeta_{R} o_{R}: Q(R) \rightarrow C(R)$ is a bijection and its inverse is the bijection $o_{R}^{-1} \eta_{R}: C(R) \rightarrow Q(R)$. Moreover $\left(o_{R}^{-1} \eta_{R}\right)(I)=R_{\nu}$.

(III) The product of any finite number of members of $\bar{C}(R)$ is again a member of $\bar{C}(R)$. Every $I \in \bar{C}(R)$ has a unique factorization

$$
I=\widehat{I} \prod_{J \in C(R)} J^{u(I, J)}
$$

with nonzero principal ideal $\widehat{I}$ in $R$, where $u(I, J) \in \mathbb{N}$ with $u(I, J)=0$ for all except finitely many $J$.

(IV) In the situation of (III), upon letting

$$
\bar{\eta}_{R}(I)=\left\{\eta_{R}(J): J \in C(R) \text { with } u(I, J)>0\right\},
$$

we have that $\bar{\eta}_{R}(I)$ is a finite subset of $D(R)^{\Delta}$ and $\mathfrak{W}(R, I)_{1}^{\Delta}=\bar{\eta}_{R}(I)$. Conversely, for any finite subset $U$ of $D(R)^{\Delta}$, upon letting

$$
\bar{\zeta}_{R}(U)=\prod_{V \in U} \zeta_{R}(V)
$$

we have $\bar{\zeta}_{R}(U) \in \bar{C}(R)$ and $\mathfrak{W}\left(R, \bar{\zeta}_{R}(U)\right)_{1}^{\Delta}=U$.

\section{Answers TO DICRITICAL QUESTIONS}

Let $R$ be a two dimensional regular local domain with quotient field $L$. Let $(x, y)$ be generators of $M(R)$ and let $K=H(R)=R / M(R)$ where we recall that $H_{R}: R \rightarrow H(R)=R / M(R)$ is the residue class epimorphism.

In Section 2 of [Ab7] we have introduced the sets $\mathfrak{B}(R, J), \mathfrak{B}(R, J)^{\sharp}, \mathfrak{B}(R, J)^{b}$, and $\mathfrak{Q}(R, J)$. We shall answer (1.1) mostly in terms of the sets $\mathfrak{B}(R, J)$ and $\mathfrak{Q}(R, J) \backslash \mathfrak{B}(R, J)$ of big and small stars of the pencil $J=J_{R}(z)$. In terms of the sharp and flat star sets $\mathfrak{B}(R, J)^{\sharp}$ and $\mathfrak{B}(R, J)^{b}$ of $J$ in $R$, Proposition 2.1 can be partly paraphrased by saying that if $R / M(R)$ is infinite and $U \subset D(R)^{\Delta}$ is finite, then $\mathfrak{B}(R, J)^{\sharp}=\mathfrak{B}(R, J)^{b}=\mathfrak{B}(R, J)=U$ for some $J=(a, b) R$ with $a \neq 0 \neq b$ in $M(R)$. Likewise Proposition 2.2 can be paraphrased by saying that if $J$ is special at $R$, then $\mathfrak{B}(R, J)^{b}=\mathfrak{B}(R, J)$. 
We shall write the answers to (1.1) as a series of propositions. Out of them, (3.2) will be obtained by dissecting and reassembling the proof of 2.2 . These Propositions 3.1 to 3.5 are a continuation of Propositions 4.1 to 4.3 of [Ab7].

Proposition 3.1. As facts which may be used tacitly, let us note that if $R^{\prime} \in$ $Q_{1}(R)$ with $M(R) R^{\prime}=x R^{\prime}$, then we have (1) to (4). The proofs of (1) to (3) are straightforward. To prove (4), use induction on $m$.

(1) $\zeta \in R^{\prime} \Rightarrow \epsilon \zeta \in R[y / x]$ for some $\epsilon \in R[y / x] \cap U\left(R^{\prime}\right)$.

(2) $\zeta \in R[y / x] \Rightarrow \zeta x^{n} \in R$ for some $n \in \mathbb{N}$.

(3) $\eta \in R$ with $\operatorname{ord}_{R} \eta=n \in \mathbb{N} \Rightarrow \eta / x^{n} \in R^{\prime}$ with $\operatorname{ord}_{R^{\prime}}\left(\eta / x^{n}\right) \leq e$.

(4) $\xi \in M(R)^{n}$ with integers $m \geq n \geq 0 \Rightarrow \xi=\xi^{\prime}+\sum_{0 \leq i \leq n} \xi_{i} x^{n-i} y^{i}$ with $\xi^{\prime} \in M(R)^{m}$ and $\xi_{i} \in S$.

Proposition 3.2. Let $\left(R_{j}\right)_{0 \leq j \leq \nu}$ be the finite $Q D T$ sequence of $R$ along $V \in$ $D(R)^{\Delta}$, and let $z \in L^{\times}$generate a special pencil at $R$. Then we have the following:

(I) If $R_{\nu}$ is a big star of $J_{R}(z)$, then $z$ generates a special pencil at $R_{\nu}$.

(II) If $R$ is a big star of $J_{R}(z)$, then $z$ is residually a polynomial over $R$ relative to $o(R)$.

(III) If $R_{\nu}$ is a big star of $J_{R}(z)$, then $z$ is residually a polynomial over $R$ relative to $V$.

Proof. Since $z$ generates a special pencil at $R$, we may assume that $z=F / G$, where $F, G$ in $R^{\times}$are such that $(F, G) R$ is an $M(R)$-primary ideal in $R$ and $G R=x^{m} y^{n} R$ for some $m, n$ in $\mathbb{N}$ with $m n=0$. Clearly $J_{R}(z)=(F, G) R$. Take a coefficient set $\kappa$ of $R$ and let

$$
\left(R_{j}, x_{j}, y_{j}, \kappa_{j}\right)_{0 \leq j \leq \nu}
$$

be the finite QDT sequence of $(R, x, y, \kappa)$ along $V$. Now in view of (4.1) of $\mathrm{AbL}$, we get a proof of (I) by deleting the last sentence from the proof of (4.8) of $\mathrm{AbL}$. (II) follows from (4.1) and (4.6)(VI) of $\mathrm{AbL}$. (III) follows from (I) and (II).

Proposition 3.3. As preliminary answers to (1.1) we have the following:

(I) If $J$ is a special pencil at $R$, then $\mathfrak{B}(R, J)$ is globally unforked. In other words, adjacent to a big star of a special $J$ there is at most one other star of $J$ and it cannot be big.

(II) $\mathfrak{D}^{*}(R, U)^{\dagger}=\emptyset$ for some finite $U \subset D(R)^{\Delta}$.

Proof. Clearly (I) $\Rightarrow$ (II). In view of (4.1)(iii) of Ab7, by (3.2)(I) we get (I).

Note on (3.3). In conjunction with $(3.3)(\mathrm{II})$ we may ask if $\mathfrak{D}^{*}(R,\{V\})^{\dagger} \neq \emptyset$ for all $V \in D(R)^{\Delta}$ ? This is equivalent to asking if for every $V \in D(R)^{\Delta}$ we have $\mathfrak{D}(R, J)=\{V\}$ for some special pencil $J$ at $R$ ? In other words, looking at any spot in the firmament, can we make a special fireworks with a lone big star at that spot? In (3.4) we shall explore this while generalizing (4.3) of [Ab7].

Proposition 3.4. Let $\kappa$ be a coefficient set of $R$ and let $\left(R_{j}, x_{j}, y_{j}, \kappa_{j}\right)_{0 \leq j \leq \nu}$ be the finite $Q D T$ sequence of $(R, x, y, \kappa)$ along $V \in D(R)^{\Delta}$. We are asking if we always have $\mathfrak{D}(R, J)=\{V\}$ for some special pencil $J$ at $R$ ? To explore this we try to use induction on the depth $d(R, V)=\nu+1$. In (I) to (V) I shall generalize (4.3)(i) to $(4.3)(\mathrm{v})$ of $\mathrm{Ab7}$ respectively. In (VI) to (X) I shall start extending the matter from special pencils to semispecial pencils. The proof of Propositions 1.2 and 3.1 of Ab7. has led me to the notion of QDT sequences without localizing. This indeed 
was the missing link which removed the obstacle in the construction of $A Q D T=$ ANTIQUADRATC sequences!! I am referring to the above QDT sequence read backwards like $R_{\nu}, R_{\nu-1}, \ldots, R_{1}, R_{0}$. The obstacle was the hypothesis of $R$ having a coefficient field required in the original (unpublished) version of (4.3) of [Ab7. Let us generalize the subsets

$$
S(e, d, c), \ldots, S(e=d<c)
$$

of $S[X, Y]$ introduced in (4.3) of [Ab7]. So given any ring $S$, for any $c$ in $\mathbb{N}_{+}$let $S(c)$ be the set of all $f=f(X, Y) \in S[X, Y]$ such that

$$
\operatorname{deg}_{Y} f=c \quad \text { and } \quad f(0, Y)=Y^{c} .
$$

Equivalently, $S(c)$ is the set of all $f \in S[X, Y]$ which are of the form

$$
f(X, Y)=Y^{c}+X g(X, Y) \text { with } g=g(X, Y) \in S[X, Y] \quad \text { and } \operatorname{deg}_{Y} g \leq c .
$$

For any $d, c$ in $\mathbb{N}_{+}$let

$$
S(d, c)=\left\{f \in S(c): \operatorname{ord}_{X, Y} f(X, Y)=d\right\}
$$

and for any $d=c$ in $\mathbb{N}_{+}$let

$$
S(d=c)=\left\{f \in S(d, c): \operatorname{ord}_{X, Y}[f(X, Y)-f(0, Y)]>c\right\} .
$$

Recall that for any e,d,c in $\mathbb{N}_{+}$we have let

$$
S(e, d, c)=\left\{f \in S(d, c): \operatorname{deg}_{X} f(X, Y)<e \text { and } \operatorname{ord}_{Y} f(X, Y)>0\right\}
$$

and for any $e>d=c$ in $\mathbb{N}_{+}$we have let

$$
S(e>d=c)=\left\{f \in S(e, d, c): \operatorname{ord}_{X, Y}[f(X, Y)-f(0, Y)]>c\right\}
$$

and for any $e<d \leq c$ in $\mathbb{N}_{+}$we have let

$$
S(e<d \leq c)=\left\{f \in S(e, d, c): \operatorname{ord}_{X, Y}[f(X, Y)-f(0, Y)]>e\right\}
$$

and for any $e=d<c$ in $\mathbb{N}_{+}$we have let

$$
S(e=d<c)=\left\{f \in S(e, d, c): \operatorname{ord}_{X} \operatorname{info}_{X, Y} f(X, Y)<e\right\} .
$$

In the rest of (3.4) let $S$ be a coefficient ring of $R$ with $\kappa \subset S$; for instance $S=R$. Also assume that $R_{\nu}$ is residually rational over $R$ and note that then, for $0 \leq j \leq \nu, S$ is a coefficient ring of $R_{j}$ with $\kappa=\kappa_{j} \subset S$. For $0<j \leq \nu$ we clearly have a unique

$$
p_{j}(Z)=Z+\pi_{j} \quad \text { with } \quad \pi_{j} \in \kappa
$$

such that

$$
y_{j}=p_{j}\left(y_{j-1} / x_{j-1}\right) \quad \text { or } \quad x_{j}=p_{j}\left(x_{j-1} / y_{j-1}\right)
$$

according as $x_{j}=x_{j-1}$ or $x_{j} \neq x_{j-1}$; note that in the latter case we have $\pi_{j}=0$.

(I) The statement and the proof are the same as (4.3)(i) of Ab7.

(II) Assume that $\nu>0$ with $x_{1}=x$. Consider the special primary pencil $J_{1}=$ $\left(a_{1}, b_{1}\right) R_{1}$ at $R_{1}$ given by $b_{1}=x_{1}^{e}$ and $a_{1}=f_{1}\left(x_{1}, y_{1}\right)$ with $e \in \mathbb{N}_{+}$and $f_{1} \in S(c)$. Let $f(X, Y)=X^{c} f_{1}\left(X, p_{1}(Y / X)\right)$ and $d=c$. Then $f \in S(d=c)$ and for the special primary pencil $J=(a, b) R$ at $R$ given by $b=x^{e+c}$ and $a=f(x, y)$ we have $\left(R, R_{1}\right)(J)=J_{1}$ and $R$ is a unifurcated small star of $J$. Moreover, if $\pi_{1}=0$ and $f_{1} \in S\left(e_{1}, d_{1}, c_{1}\right)$ with $\left(e_{1}, d_{1}, c_{1}\right)=(e, d, c)$, then $f \in S(e+c>d=c)$. 
Proof. Clearly $f_{1} \in S[X, Y]$ belongs to $S(c)$ iff

$$
f_{1}(X, Y)=Y^{c}+\sum_{i>0 \text { and } 0 \leq j \leq c} \alpha_{i j} X^{i} Y^{j} \quad \text { with } \quad \alpha_{i j} \in S .
$$

Since $f(X, Y)=X^{c} f_{1}\left(X, p_{1}(Y / X)\right)$ we get

$$
f(X, Y)=\left(Y+\pi_{1} X\right)^{c}+\sum_{i>0 \text { and } 0 \leq j \leq c} \alpha_{i j} X^{i+c-j}\left(Y+\pi_{1} X\right)^{j} .
$$

It follows that $f \in S(d=c)$ and for the special primary pencil $J=(a, b) R$ at $R$ given by $b=x^{e+c}$ and $a=f(x, y)$ we have $\left(R, R_{1}\right)(J)=J_{1}$. By (4.1) of Ab7. we see that $R$ is a unifurcated small star of $J$. By the above two displayed equations we also see that if $\pi_{1}=0$ and $f_{1} \in S\left(e_{1}, d_{1}, c_{1}\right)$ with $\left(e_{1}, d_{1}, c_{1}\right)=(e, d, c)$, then $f \in S(e+c>d=c)$.

(III) Assume that $\nu>0$ with $x_{1} \neq x$. Consider the special primary pencil $J_{1}=\left(a_{1}, b_{1}\right) R_{1}$ at $R_{1}$ given by $b_{1}=x_{1}^{e}$ and $a_{1}=f_{1}\left(x_{1}, y_{1}\right)$ with $f_{1} \in S(e, d, c)$. Let $f(X, Y)=Y^{e} f_{1}\left(p_{1}(X / Y), Y\right)$. Then $f(X, Y) \in S[X, Y]$ and upon letting $d^{*}=\operatorname{ord}_{X, Y} f(X, Y)$ we have that: $e<d^{*} \leq e+c$ and $f \in S\left(e<d^{*} \leq e+c\right)$ and for the special primary pencil $J=(a, b) R$ at $R$ given by $b=x^{e}$ and $a=f(x, y)$ we have $\left(R, R_{1}\right)(J)=J_{1}$ and $R$ is a unifurcated small star of $J$.

Proof. In the proof of (4.3)(iii) of [Ab7] change $f^{\prime \prime}$ to $f_{1}$.

(IV) Assume that $\nu>0$ with $x_{1} \neq x$. Consider the generators $(u, v)$ of $M\left(R_{1}\right)$ given by $(u, v)=\left(x_{1}, y_{1}+x_{1}\right)$. Consider the special primary pencil $J_{1}=\left(a_{1}, b_{1}\right) R_{1}$ at $R_{1}$ given by $b_{1}=u^{e}$ and $a_{1}=f_{1}(u, v)$ with $f_{1} \in S(e>d=c)$. Then there exists $f \in S(e=e<e+c)$ such that for the special primary pencil $J=(a, b) R$ at $R$ given by $b=x^{e}$ and $a=f(x, y)$ we have $\left(R, R_{1}\right)(J)=J_{1}$ and $R$ is a unifurcated big star of $J$. Actually we may take $f$ to be given by the explicit formula

$$
f(X, Y)=Y^{e} \widehat{f}(X / Y, Y), \text { where } \widehat{f}(X, Y)=\tau_{e} f_{1}(X, Y+X) .
$$

Proof. In the proof of (4.3)(iv) of [Ab7 change $f^{\prime \prime}$ to $f_{1}$.

(V) The statement and the proof are the same as (4.3)(v) of [Ab7. Note that in item $(\mathrm{V})$ we are suspending the first sentence "Let $\kappa \ldots V \in D(R)^{\Delta}$ " of the preamble of (3.4).

(VI) Let $\nu=3$. Consider the special pencil $J$ at $R$ and the semispecial small terminal tower $\left(R_{j}, J_{j}\right)_{0 \leq j \leq 3}$ at $R$ with $J=J_{0}$ given by

$$
\left\{\begin{array}{r}
J_{0}=\left(a_{0}, b_{0}\right) R_{0} \text { with }\left(b_{0}, a_{0}\right)=\left(x_{0}^{7},\left(y_{0}^{2}+\pi x_{0}^{3}\right)^{2}\right), \\
J_{1}=\left(a_{1}, b_{1}\right) R_{1} \text { with }\left(b_{1}, a_{1}\right)=\left(x_{1}^{3},\left(y_{1}^{2}+\pi x_{1}\right)^{2}\right) \text { and }\left(x_{1}, y_{1}\right)=\left(x_{0}, y_{0} / x_{0}\right), \\
J_{2}=\left(a_{2}, b_{2}\right) R_{2} \text { with }\left(b_{2}, a_{2}\right)=\left(x_{2}^{3} y_{2},\left(y_{2}+\pi x_{2}\right)^{2}\right) \text { and }\left(x_{2}, y_{2}\right)=\left(x_{1} / y_{1}, y_{1}\right), \\
J_{3}=\left(a_{3}, b_{3}\right) R_{3} \text { with }\left(b_{3}, a_{3}\right)=\left(x_{3}^{2}, y_{3}^{2}\right) \text { and }\left(x_{3}, y_{3}\right)=\left(x_{2},\left(y_{2} / x_{2}\right)+\pi\right) \\
\quad \text { where } 0 \neq \pi=\pi_{3} \in \kappa .
\end{array}\right.
$$

As a supplement to the Note on (3.3), this shows the existence of $V \in D(R)^{\Delta}$ with $d(R, V)=4$ such that $\mathfrak{D}(R, J)^{b}=\mathfrak{D}(R, J)=\{V\}$ for some special $J$ but, as we shall prove, (1) $\mathfrak{D}(R, \widehat{J})^{\sharp} \neq\{V\}$ for all special $\widehat{J}$. Moreover we shall also prove that (2) $\operatorname{ord}_{V} x=2$ and $\operatorname{ord}_{V} y=3$ with $\operatorname{ord}_{V}\left(y^{2}+\pi x^{3}\right)=7$. Furthermore, upon letting $I=\zeta_{R}(V)$, where $\zeta_{R}$ is the Zariski map described in Proposition 2.4 of Section 2, we shall prove that (3) $I=\left(y^{2}+\pi x^{3}, x^{4}, x^{2} y\right) R$ and $\operatorname{ord}_{V} I=7$. 
Proof. By definition we have $\operatorname{ord}_{V} x_{3}=1=\operatorname{ord}_{V} y_{3}$. Now the equation $\left(x_{3}, y_{3}\right)=$ $\left(x_{2},\left(y_{2} / x_{2}\right)+\pi\right)$ where $0 \neq \pi=\pi_{3} \in \kappa$ yields $\operatorname{ord}_{V} x_{2}=\operatorname{ord}_{V} y_{2}=1$ and $\operatorname{ord}_{V}\left(y_{2}+\right.$ $\left.\pi x_{2}\right)=2$. Hence the equation $\left(x_{2}, y_{2}\right)=\left(x_{1} / y_{1}, y_{1}\right) \operatorname{gives} \operatorname{ord}_{V} x_{1}=2$ and $\operatorname{ord}_{V} y_{1}=$ 1 with $\operatorname{ord}_{V}\left(y_{1}^{2}+\pi x_{1}\right)=3$. Now the equation $\left(x_{1}, y_{1}\right)=\left(x_{0}, y_{0} / x_{0}\right)$ yields $\operatorname{ord}_{V} x_{0}=$ 2 and $\operatorname{ord}_{V} y_{0}=3$ with $\operatorname{ord}_{V}\left(y_{0}^{2}+\pi x_{0}^{3}\right)=7$. This proves $(2)$.

Since we have a tower with $J_{3}=\left(x_{3}^{2}, y_{3}^{2}\right) R_{3}$ and $J=\left(x^{7},\left(y^{2}+\pi x^{3}\right)^{2}\right) R$, by $(2.4)$ it follows that $\operatorname{ord}_{V} I^{2}=14$ and hence $\operatorname{ord}_{V} I=7$. Upon letting

$$
E=\left\{\operatorname{ord}_{V} z: z \in M(R) \backslash M(R)^{2}\right\},
$$

by (2) we get $E=\{2,3\}$; if $\mathfrak{D}(R, \widehat{J})^{\sharp}=\{V\}$ for a special $\widehat{J}$, then we must have $\operatorname{ord}_{V} z^{p}=\operatorname{ord}_{V} I=7$ for some $z \in M(R) \backslash M(R)^{2}$ and $p \in \mathbb{N}_{+}$; this proves (1). It only remains to show that $R \cap M(V)^{7}=\left(y^{2}+\pi x^{3}, x^{4}, x^{2} y\right) R$. By (2) we get RHS $\subset$ LHS. By (2) we shall show that any $z$ in LHS can be written as $z=u\left(y^{2}+\pi x^{3}\right)+v x^{2} y+w x^{4}$ with $u, v, w$ in $R$, and this will complete the proof.

To see this, clearly any $z$ in $R$ can be written as $z=u\left(y^{2}+\pi x^{3}\right)+v^{\prime} y+w^{\prime}$ with $u, v^{\prime}, w^{\prime}$ in $R$; for instance take $u=v^{\prime}=0$ and $w^{\prime}=z$. Assuming $\operatorname{ord}_{V} z>0$, we must have $w^{\prime} \in M(R)$ and hence $w^{\prime}=v_{1} y+w_{1} x$ with $v_{1}, w_{1}$ in $R$; absorbing $v_{1}$ in $v^{\prime}$ we get $z=u\left(y^{2}+\pi x^{3}\right)+v^{\prime} y+w_{1} x$ with $u, v^{\prime}, w_{1}$ in $R$. Now assuming $\operatorname{ord}_{V} z>2$ we see that $w_{1} \in M(R)$; hence $w_{1}=v_{2} y+w_{2} x$ with $v_{2}, w_{2}$ in $R$. Absorbing $v_{2}$ in $v^{\prime}$ we get $z=u\left(y^{2}+\pi x^{3}\right)+v^{\prime} y+w_{2} x^{2}$ with $u, v^{\prime}, w_{2}$ in $R$. Now in turn assuming $\operatorname{ord}_{V} z>3$ we see that $v^{\prime} \in M(R)$ and hence $v^{\prime}=u^{\prime \prime} y+v^{\prime \prime} x$ with $u^{\prime \prime}, v^{\prime \prime}$ in $R$. Absorbing $u^{\prime \prime}$ in $u$ we get $z=u\left(y^{2}+\pi x^{3}\right)+v^{\prime \prime} x y+w_{2} x^{2}$ with $u, v^{\prime \prime}, w_{2}$ in $R$. Next assuming $\operatorname{ord}_{V} z>4$ we get $w_{2} \in M(R)$ and hence $w_{2}=v_{3} y+w_{3} x$ with $v_{3}, w_{3}$ in $R$. Absorbing $v_{3} x$ in $v^{\prime \prime}$ we get $z=u\left(y^{2}+\pi x^{3}\right)+v^{\prime \prime} x y+w_{3} x^{3}$ with $u, v^{\prime \prime}, w_{3}$ in $R$. Now assuming $\operatorname{ord}_{V} z>5$ we get $v^{\prime \prime} \in M(R)$ and hence $v^{\prime \prime}=u^{*} y+v x$ with $u^{*}, v$ in $R$. Absorbing $u^{*} x$ in $u$ we get $z=u\left(y^{2}+\pi x^{3}\right)+v x^{2} y+w_{3} x^{3}$ with $u, v, w_{3}$ in $R$. Finally assuming $\operatorname{ord}_{V} z>6$ we get $w_{3} \in M(R)$; hence $w_{3}=v_{4} y+w x$ with $v_{4}, w$ in $R$. Absorbing $v_{4} x$ in $v$ we get $z=u\left(y^{2}+\pi x^{3}\right)+v x^{2} y+w x^{4}$ with $u, v, w$ in $R$.

(VII) In contrast to item (VI)(1), let us show that if $\operatorname{ord}_{R} \zeta_{R}(V)=1$, then $\mathfrak{D}(R, J)^{\sharp}=\{V\}$ for some special pencil $J$ at $R$. Note that in the above example we have $\operatorname{ord}_{R} \zeta_{R}(V)=2$. Upon letting

$$
E=\left\{\operatorname{ord}_{V} z: z \in M(R) \backslash M(R)^{2}\right\}
$$

with

$$
m=\min (E) \quad \text { and } \quad n=\max (E)
$$

and

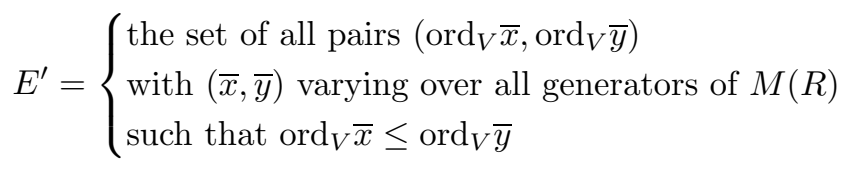

we shall show that

$$
\left\{\begin{array}{l}
\operatorname{ord}_{R} \zeta_{R}(V)=1 \\
\Leftrightarrow\left(R_{j}\right)_{0 \leq j \leq \nu} \text { is a pillar at }(R, \bar{x}) \text { for some } \bar{x} \in M(R) \backslash M(R)^{2} \\
\Leftrightarrow m=1 \\
\Leftrightarrow E \subset m \mathbb{Z}
\end{array}\right.
$$


and

$$
\left\{\begin{array}{l}
\operatorname{ord}_{R} \zeta_{R}(V)=1 \\
\Rightarrow\left(R_{j}, \zeta_{R_{j}}(V)\right)_{0 \leq j \leq \nu} \text { is a special tower at } R \\
\quad \text { and } \zeta_{R}(V) \text { is a special pencil at } R \\
\quad \text { and } \mathfrak{D}\left(R, \zeta_{R}(V)\right)^{\sharp}=\{V\} \text { with }(m, n)=(1, \nu+1), \\
\quad \text { and if } \nu=0, \text { then } \zeta_{R}(V)=M(R), \\
\quad \text { whereas if } \nu>0, \text { then } E^{\prime}=\{(1,1),(1,2), \ldots,(1, n)\} \text { and } \\
\text { for all } \bar{x}, \bar{y} \text { in } M(R) \backslash M(R)^{2} \text { with }\left(\operatorname{ord}_{V} \bar{x}, \operatorname{ord}_{V} \bar{y}\right)=(1, n) \\
\text { we have } \zeta_{R}(V)=\left(\bar{x}^{n}, \bar{y}\right) R \text { with } M(R)=(\bar{x}, \bar{y}) R .
\end{array}\right.
$$

In greater detail let us prove the following:

(i) Assume that $\left(R_{j}\right)_{0 \leq j \leq \nu}$ is a pillar at $(R, x)$, i.e., $x=x_{j}$ for $0 \leq j \leq \nu$. Consider the special primary pencil $J_{\nu}=\left(a_{\nu}, b_{\nu}\right) R_{\nu}$ at $R_{\nu}$ given by $b_{\nu}=x_{\nu}^{e_{\nu}}$ and $a_{\nu}=f_{\nu}\left(x_{\nu}, y_{\nu}\right)$ with $e_{\nu} \in \mathbb{N}_{+}$and $f_{\nu} \in S(c)$. Let $d=c$ and for $0 \leq j<\nu$ let

$$
e_{j}=e_{\nu}+(\nu-j) c
$$

and for $0 \leq j<\nu$ let us reverse inductively by putting

$$
f_{j}(X, Y)=X^{c} f_{j+1}\left(X, p_{j+1}(Y / X)\right) .
$$

Then for $0 \leq j<\nu: f_{j} \in S(d=c)$, for the special primary pencil $J_{j}=\left(a_{j}, b_{j}\right) R_{j}$ at $R_{j}$ given by $\left(b_{j}, a_{j}\right)=\left(x_{j}^{e_{j}}, f_{j}\left(x_{j}, y_{j}\right)\right)$ we have $\left(R_{j}, R_{j+1}\right)\left(J_{j}\right)=J_{j+1}$, and $R_{j}$ is a unifurcated small star of $J_{j}$. In other words, $\left(R_{j}, J_{j}\right)_{0 \leq j \leq \nu}$ is a special small tower at $R$.

(ii) Let the hypothesis be as in (i) and assume that $\left(b_{\nu}, a_{\nu}\right)=\left(x_{\nu}, y_{\nu}\right)$ with $\left(e_{\nu}, c, f_{\nu}(X, Y)\right)=(1,1, Y)$. Then $\left(R_{j}, J_{j}\right)_{0 \leq j \leq \nu}$ is a special terminal small tower at $R$, and for $0 \leq j \leq \nu$ we have

$$
\mathfrak{D}\left(R_{j}, J_{j}\right)^{\sharp}=\{V\}
$$

and

$$
\operatorname{ord}_{R_{j}} \zeta_{R_{j}}(V)=1=\operatorname{ord}_{V} x_{j} \text { with } \operatorname{ord}_{V} \zeta_{R_{j}}(V)=e_{j}=\nu+1-j
$$

and

$$
\zeta_{R_{j}}(V)=J_{j}=\left(a_{j}, b_{j}\right) R_{j} \text { with } b_{j}=x_{j}^{e_{j}} \text { and } a_{j}=y_{j}+\sum_{j<i \leq \nu} \pi_{i} x^{i-j} .
$$

(iii) Conversely, assume that $\operatorname{ord}_{R} \zeta_{R}(V)=1$. Then we can find generators $(\bar{x}, \bar{y})$ of $M(R)$ such that $\bar{y} \in \zeta_{R}(V)$. Moreover, for any such $(\bar{x}, \bar{y})$ we have that $\left(R_{j}\right)_{0 \leq j \leq \nu}$ is a pillar at $(R, \bar{x})$.

(iv) Concerning $m$ and $n$ we have the following:

(A) Let $\bar{x}, \bar{y}$ in $M(R) \backslash M(R)^{2}$ be such that $\operatorname{ord}_{V} \bar{x}=m<\operatorname{ord}_{V} \bar{y}$. Then we have $M(R)=(\bar{x}, \bar{y}) R$ and $\operatorname{ord}_{V} \bar{y} \leq m \nu+1$.

(B) $n \leq m \nu+1$.

(C) Assume $\nu=0$. Then $m=n=1$ and $\zeta_{R}(V)=M(R)$.

(D) Assume $\nu>0$ and $m=1$. Then $n=\nu+1$ and

$$
\left.E^{\prime}=\{(1,1),(1,2), \ldots,(1, n))\right\} .
$$

Moreover, for all $\bar{x}, \bar{y}$ in $M(R) \backslash M(R)^{2}$ with $\left(\operatorname{ord}_{V} \bar{x}, \operatorname{ord}_{V} \bar{y}\right)=(1, n)$ we have $\zeta_{R}(V)=\left(\bar{x}^{n}, \bar{y}\right) R$ with $M(R)=(\bar{x}, \bar{y}) R$. 
(E) Assume $m>1$. Then $\nu>1$ and $\left(R_{j}\right)_{0 \leq j \leq \nu}$ is not a pillar at $(R, \bar{x})$ for any $\bar{x} \in M(R) \backslash M(R)^{2}$.

(F) Assume $m>1$. Then, given any generators $(\bar{x}, \bar{y})$ of $M(R)$ with

$$
\operatorname{ord}_{V} \bar{x}=m \text { and } \operatorname{ord}_{V} \bar{y}=e m \text { for some } e \in \mathbb{N}_{+},
$$

we have $\operatorname{ord}_{V}\left(\bar{y}-\pi \bar{x}^{e}\right)>\operatorname{ord}_{V} \bar{y}$ for some $\pi \in \kappa$.

(G) Assume $m>1$. Then $m<n \in\left(\mathbb{N}_{+} \backslash(m \mathbb{Z})\right)$ and

$$
E^{\prime}=\{(m, m),(m, 2 m), \ldots,(m, d m),(m, n)\},
$$

where $d$ is the largest integer $<n / m$.

Proof of $(i)$. Inductively use (II).

Proof of (ii). We get (1) because the pulling down is unifurcated. Claim (3) except the first equality follows from the iterative definition of the $f_{j}$. In particular $\operatorname{ord}_{R_{J}} J_{j}=1$ and hence $J_{j}$ is a simple complete ideal. Now the proper transform of $J_{j}$ in $R_{\nu}$ is $M\left(R_{\nu}\right)$, i.e., $\left(R_{j}, R_{\nu}\right)\left(J_{j}\right)=M\left(R_{\nu}\right)$. Therefore we get $\zeta_{R_{j}}(V)=J_{j}$. Iteratively we also see that $\operatorname{ord}_{V} x_{j}=1$ and $\operatorname{ord}_{V} J_{j}=e_{j}$. This proves (2) and (3).

Proof of $\left(\right.$ iii). Since $\operatorname{ord}_{R} \zeta_{R}(V)=1$, there exist generators $(\bar{x}, \bar{y})$ of $M(R)$ with $\bar{y} \in \zeta_{R}(V)$. For any such generators, the image of $\bar{x}$ generates the maximal ideal of the one dimensional regular local domain $R / \bar{y} R$, and therefore upon letting $e=\operatorname{ord}_{(R / \bar{y} R)} \zeta_{R}(V)$ we see that $e$ is a positive integer with $\zeta_{R}(V)=\left(\bar{x}^{e}, \bar{y}\right) R$. Now, assuming $\zeta_{R}(V)=\left(\bar{x}^{e}, \bar{y}\right) R$, by induction on $\nu$ let us prove that $e=\nu+1$ and $\left(R_{j}\right)_{0 \leq j \leq \nu}$ is a pillar at $(R, \bar{x})$. This is obviously true for $\nu=0$ because then $\zeta_{R}(V)=M(R)$. So let $\nu>0$ and assume it is true for $\nu-1$. Now $\nu>0$ implies that $\zeta_{R}(V) \neq M(R)$ but $\zeta_{R}(V)$ goes through $R_{1}$. Therefore $e>1$ with $M(R) R_{1}=\bar{x} R_{1}$ and $\zeta_{R_{1}}(V)=\left(R, R_{1}\right)\left(\zeta_{R}(V)\right)=\left(\bar{x}^{e-1}, \bar{y} / \bar{x}\right) R_{1}$ with $M\left(R_{1}\right)=(\bar{x}, \bar{y} / \bar{x})$. Therefore by the induction hypothesis $e-1=(\nu-1)+1$ and $\left(R_{j}\right)_{1 \leq j \leq \nu}$ is a pillar at $\left(R_{1}, \bar{x}\right)$. Consequently $e=\nu+1$ and $\left(R_{j}\right)_{0 \leq j \leq \nu}$ is a pillar at $(R, \bar{x})$.

Proof of $(i v)(A)$. For some $\widehat{y} \in M(R) \backslash M(R)^{2}$ we have $M(R)=(\bar{x}, \widehat{y}) R$ and hence $\bar{y}=u \bar{x}+v \widehat{y}$ for some $u, v$ in $R$. Suppose if possible that $v \in M(R)$; then, because $\bar{y} \notin$ $M(R)^{2}$, we must have $u \notin M(R)$ and hence $\operatorname{ord}_{V} \bar{y}=m$, which is a contradiction. Therefore $v \notin M(R)$ and hence $M(R)=(\bar{x}, \bar{y}) R$.

Now suppose if possible that $\operatorname{ord}_{V} \bar{y}>m \nu+1$. Then, because $V=o\left(R_{\nu}\right)$, by induction on $j$ we get $M\left(R_{j}\right)=\left(\bar{x}, \bar{y} / \bar{x}^{j}\right) R_{j}$ for $0 \leq j \leq \nu$ and hence in particular $M\left(R_{\nu}\right)=\left(\bar{x}, \bar{y} / \bar{x}^{\nu}\right) R_{\nu}$. This tells us that $\operatorname{ord}_{V} \bar{x}=1=\operatorname{ord}_{V}\left(\bar{y} / \bar{x}^{\nu}\right)$ and therefore $\operatorname{ord}_{V} \bar{y}=m \nu+1$, which is a contradiction. Hence we must have $\operatorname{ord}_{V} \bar{y} \leq m \nu+1$.

Proof of $(i v)(B)$. This follows from (iv)(A) and (iv)(C).

Proof of $(i v)(C)$. This is obvious.

Proof of $(i v)(D)$. Given any $\bar{x} \in M(R) \backslash M(R)^{2}$ with $\operatorname{ord}_{V} \bar{x}=1$ we can write $M(R)=(\bar{x}, \widetilde{y}) R$ with $\widetilde{y} \in M(R) \backslash M(R)^{2}$. Now $\left(R_{j}\right)_{0 \leq j \leq \nu}$ is a pillar at $(R, \bar{x})$ and hence, in view of (iv)(A), by (ii) we see that $n=\nu+1$, and there exists $\widehat{y} \in \zeta_{R}(V)$ with $\operatorname{ord}_{V} \widehat{y}=n$ such that

$$
\zeta_{R}(V)=\left(\bar{x}^{n}, \widehat{y}\right) R
$$


and

$$
M(R)=(\bar{x}, \widehat{y}) R .
$$

Now for $1 \leq i<n$ we have $M(R)=\left(\bar{x}, \widehat{y}+\bar{x}^{i}\right)$ with $\left(\operatorname{ord}_{V} \bar{x}, \operatorname{ord}_{V}\left(\widehat{y}+\bar{x}^{i}\right)\right)=(1, i)$ and hence we conclude that $\left.E^{\prime}=\{(1,1),(1,2), \ldots,(1, n))\right\}$.

It only remains to show that, given any $\bar{y} \in M(R) \backslash M(R)^{2}$ with $\operatorname{ord}_{V} \bar{y}=n$, we have $\zeta_{R}(V)=\left(\bar{x}^{n}, \bar{y}\right) R$ with $M(R)=(\bar{x}, \bar{y}) R$. By (iv)(A) we get

$$
M(R)=(\bar{x}, \bar{y}) R
$$

and we clearly have

$$
\operatorname{ord}_{V} \bar{x}=1 \text { and } \operatorname{ord}_{V} \bar{y}=\operatorname{ord}_{V} \widehat{y}=n=\nu+1 .
$$

To prove $\zeta_{R}(V)=\left(\bar{x}^{n}, \bar{y}\right) R$, by induction on $e$ let us show that for any nonnegative integer $e \leq n$ we have $\bar{y}=u_{e} \bar{x}^{e}+v_{e} \widehat{y}$ with $u_{e}, v_{e}$ in $R$. If $e=0$, then it suffices to take $u_{e}=\bar{y}$ and $v_{e}=0$. So now let $0<e<n$ and assume $\bar{y}=u_{e-1} \bar{x}^{e-1}+v_{e-1} \widehat{y}$ with $u_{e-1}, v_{e-1}$ in $R$. If $u_{e-1} \notin M(R)$, then $\operatorname{ord}_{V} \bar{y}=e-1<n$, which is a contradiction. Therefore $u_{e-1} \in M(R)$ and hence $u_{e-1}=u_{e} \bar{x}+v \widehat{y}$. Upon letting $v_{e}=v_{e-1}+v \bar{x}^{e-1}$ we get $\bar{y}=u_{e} \bar{x}^{e}+v_{e} \widehat{y}$ with $u_{e}, v_{e}$ in $R$. This completes the induction. Taking $e=n$ we obtain

$$
\bar{y}=u_{n} \bar{x}^{n}+v_{n} \widehat{y} \text { with } u_{n}, v_{n} \text { in } R .
$$

In view of (2) to (5), by induction on $j$ we see that for $0 \leq j \leq \nu$ we have

$$
\left(\bar{x}, \bar{y} / \bar{x}^{j}\right) R_{j}=M\left(R_{j}\right)
$$

and

$$
\bar{y} / \bar{x}^{j}=u_{n} \bar{x}^{n-j}+v_{n}\left(\widehat{y} / \bar{x}^{j}\right) \text { with } u_{n}, v_{n} \text { in } R .
$$

Taking $j=\nu$ in (6) and (7) we see that $v_{n} \notin M(R)$, and hence by (1) and (7), we get $\zeta_{R}(V)=\left(\bar{x}^{n}, \bar{y}\right) R$.

Proof of $(i v)(E)$. By (ii) we see that $\left(R_{j}\right)_{0 \leq j \leq \nu}$ is not a pillar at $(R, \bar{x})$ for any $\bar{x} \in M(R) \backslash M(R)^{2}$, and hence we must have $\nu>1$.

Proof of $(i v)(F)$. Let us prove this by induction on $\nu$. For $\nu<2$ it is vacuously true by (iv)(E). So let $\nu \geq 2$ and assume it is true for $\nu-1$. Now $R_{1}$ is residually rational over $R$ and hence there is a unique $\epsilon \in \kappa$ such that $M\left(R_{1}\right)=\left(\bar{x}, \bar{y}_{1}\right) R_{1}$ where $\bar{y}_{1}=(\bar{y}-\epsilon \bar{x}) / \bar{x}$. If $e=1$, then we are done by taking $\pi=\epsilon$. Now suppose that $e>1$. Then we must have $\epsilon=0$. Applying the induction hypothesis to the QDT sequence $\left(R_{j}\right)_{1 \leq j \leq \nu}$ and the generators $\left(\bar{x}, \bar{y}_{1}\right)$ of $M\left(R_{1}\right)$, we find $\pi \in \kappa$ such that $\operatorname{ord}_{V}\left(\bar{y}_{1}-\pi \bar{x}^{e-1}\right)>\operatorname{ord}_{V} \bar{y}_{1}$. Now $\operatorname{ord}_{V}\left(\bar{y}-\pi \bar{x}^{e}\right)>\operatorname{ord}_{V} \bar{y}$, which completes the induction.

Proof of $(i v)(G)$. Taking generators $(\bar{x}, \bar{y})$ of $M(R)$ with $\operatorname{ord}_{V} \bar{x}=m$, upon subtracting $\pi \bar{x}$ from $\bar{y}$ with suitable $\pi \in \kappa$, by (iv)(F) we can arrange that $\operatorname{ord}_{V} \bar{y}>m$. Therefore by (iv)(B) we see that $m<n \in \mathbb{N}_{+}$. If $n \in m \mathbb{Z}$, then subtracting $\pi \bar{x}^{(n / m)}$ with suitable $\pi \in \kappa$, again by (iv)(F) we can arrange that $\operatorname{ord}_{V} \bar{y}>n$, which would be a contradiction. Consequently $m<n \in\left(\mathbb{N}_{+} \backslash(m \mathbb{Z})\right)$. Now letting $d$ be the largest integer $<n / m$, for $1 \leq i \leq d$ we have $M(R)=\left(\bar{x}, \bar{y}+\bar{x}^{i}\right)$ with $\left(\operatorname{ord}_{V} \bar{x}, \operatorname{ord}_{V}\left(\bar{y}+\bar{x}^{i}\right)\right)=(m, i m)$.

In view of (iv)(A), it only remains to show that, given any $\widehat{y} \in M(R)$ with $M(R)=(\bar{x}, \widehat{y}) R$ such that $m<\operatorname{ord}_{V} \widehat{y}=q<n$ for some $q \in \mathbb{N}_{+}$, upon letting $p$ be the largest integer $\leq q / m$, we have $p m=q$. By induction on $e$ let us show 
that for any nonnegative integer $e \leq p$ we have $\bar{y}=u_{e} \bar{x}^{e}+v_{e} \widehat{y}$ with $u_{e}, v_{e}$ in $R$. If $e=0$, then it suffices to take $u_{e}=\bar{y}$ and $v_{e}=0$. So now let $0<e \leq p$ and assume $\bar{y}=u_{e-1} \bar{x}^{e-1}+v_{e-1} \widehat{y}$ with $u_{e-1}, v_{e-1}$ in $R$. If $u_{e-1} \notin M(R)$, then $\operatorname{ord}_{V} \bar{y}=e m-m<n$, which is a contradiction. Therefore $u_{e-1} \in M(R)$ and hence $u_{e-1}=u_{e} \bar{x}+v \widehat{y}$. Upon letting $v_{e}=v_{e-1}+v \bar{x}^{e-1}$ we get $\bar{y}=u_{e} \bar{x}^{e}+v_{e} \widehat{y}$ with $u_{e}, v_{e}$ in $R$. This completes the induction. Taking $e=p$ we obtain

$$
\bar{y}=u_{p} \bar{x}^{p}+v_{p} \widehat{y} \quad \text { with } u_{p}, v_{p} \text { in } R \text {. }
$$

Recall that

$$
(\bar{x}, \bar{y}) R=M(R)=(\bar{x}, \widehat{y}) R .
$$

Suppose if possible that $p m \neq q$. Then we must have $p m<q$. Now if $u_{p} \notin M(R)$, then by (1) we get $\operatorname{ord}_{V} \bar{y}=p m<n$, which is a contradiction. Therefore $u_{p} \in M(R)$ and hence by (2) we get $v_{p} \notin M(R)$. Consequently by (1) we get $\operatorname{ord}_{V} \bar{y}=q<n$, which is again a contradiction. Therefore $p m=q$. This completes the proof.

Proof of $(\dagger)$ and $(\ddagger)$. This follows from (ii) to (iv).

(VIII) In some sense "by inserting an extra row in (VI)" we get the special pencil $J$ at $R$ and the special terminal small tower $\left(R_{j}, J_{j}\right)_{0 \leq j \leq 4}$ at $R$ given by

$$
\left\{\begin{array}{l}
J_{0}=\left(a_{0}, b_{0}\right) R_{0} \text { with }\left(b_{0}, a_{0}\right)=\left(x_{0}^{4}, y_{0}^{2}+\pi x_{0}^{3}\right), \\
J_{1}=\left(a_{1}, b_{1}\right) R_{1} \text { with }\left(b_{1}, a_{1}\right)=\left(x_{1}^{2}, y_{1}^{2}+\pi x_{1}\right) \text { and }\left(x_{1}, y_{1}\right)=\left(x_{0}, y_{0} / x_{0}\right), \\
J_{2}=\left(a_{2}, b_{2}\right) R_{2} \text { with }\left(b_{2}, a_{2}\right)=\left(x_{2}^{2} y_{2}, y_{2}+\pi x_{2}\right) \text { and }\left(x_{2}, y_{2}\right)=\left(x_{1} / y_{1}, y_{1}\right), \\
J_{3}=\left(a_{3}, b_{3}\right) R_{3} \text { with }\left(b_{3}, a_{3}\right)=\left(x_{3}^{2}, y_{3}\right) \text { and }\left(x_{3}, y_{3}\right)=\left(x_{2},\left(y_{2} / x_{2}\right)+\pi\right) \\
\quad \text { where } 0 \neq \pi=\pi_{3} \in \kappa, \\
J_{4}=\left(a_{4}, b_{4}\right) R_{4} \text { with }\left(b_{4}, a_{4}\right)=\left(x_{4}, y_{4}\right) \text { and }\left(x_{4}, y_{4}\right)=\left(x_{3}, y_{3} / x_{3}\right) .
\end{array}\right.
$$

(IX) As a special case of $(\mathrm{V})$ we get the special terminal tower $\left(R_{j}, J_{j}\right)_{0 \leq j \leq 4}$ studied in (4.2) of [Ab7], and cited in (4.4) of [Ab7], which can symbolically be expressed as:

$$
J_{0}=\left(X^{5},\left[X^{4} Y+X^{3} Y^{2}\right]+X^{3} Y^{3}+3 X^{2} Y^{4}+3 X Y^{6}+Y^{8}\right) \quad(X, Y) \mapsto(X Y, Y)
$$

and

$$
J_{1}=\left(X^{5},\left(X^{4}+X^{3} Y\right)+X^{3}+3 X^{2} Y+3 X Y^{2}+Y^{3}\right) \quad(X, Y) \mapsto(X, X Y-X)
$$

and

$$
J_{2}=\left(X^{2}, X Y+Y^{3}\right) \quad(X, Y) \mapsto(X Y, Y)
$$

and

$$
J_{3}=\left(X^{2}, X+Y\right) \quad(X, Y) \mapsto(X, X Y-X)
$$

and

$$
J_{4}=(X, Y)
$$

where

$$
J_{0}, J_{2}, J_{4} \text { are the big stars. }
$$

(X) As a slightly expanded version of (IX) and as another special case of (V) we get the special terminal tower $\left(R_{j}, J_{j}\right)_{0 \leq j \leq 5}$, which can symbolically be expressed as

$$
J_{0}=\left(X^{8}, X^{6} Y^{2}+X^{5} Y^{4}+X^{3} Y^{5}+3 X^{2} Y^{7}+3 X Y^{9}+Y^{11}\right) \quad(X, Y) \mapsto(X Y, Y)
$$


and

$$
J_{1}=\left(X^{8}, X^{6}+X^{5} Y+X^{3}+3 X^{2} Y+3 X Y^{2}+Y^{3}\right) \quad(X, Y) \mapsto(X, X Y-X)
$$

and

$$
J_{2}=\left(X^{5}, X^{3} Y+Y^{3}\right) \quad(X, Y) \mapsto(X, X Y)
$$

and

$$
J_{3}=\left(X^{2}, X Y+Y^{3}\right) \quad(X, Y) \mapsto(X Y, Y)
$$

and

$$
J_{4}=\left(X^{2}, X+Y\right) \quad(X, Y) \mapsto(X, X Y-X)
$$

and

$$
J_{5}=(X, Y)
$$

where

$$
J_{0}, J_{3}, J_{5} \text { are the big stars. }
$$

Note on (3.4). I electronically communicated $(3.4)(\mathrm{X})$ to Heinzer and Sathaye on 16 September 2009. It was the discovery of this tower which got the construction of the AQDT sequences cited in the preamble of Proposition 3.4 rolling and led to the production of the entire Proposition 3.4.

Proposition 3.5. For any $V, W$ in $D(R)^{\Delta}$ let $c(R, V, W)=\operatorname{ord}_{V} \zeta_{R}(W)$, i.e., $c(R, V, W)=\min \left\{\operatorname{ord}_{V} \theta: \theta \in \zeta_{R}(W)\right\}$. Let $J$ be a special primary pencil at $R$ and assume that $J=(a, b) R$, where $b=\eta^{m}$ with $\eta \in M(R) \backslash M(R)^{2}$ and $m \in \mathbb{N}_{+}$. Let $\mathfrak{D}(R, J)=U$. Then clearly there exists $n(W) \in \mathbb{N}_{+}$for all $W \in U$ such that

$$
\prod_{W \in U} \zeta_{R}(W)^{n(W)}
$$

is the integral closure of $J$ in $R$, and hence

$$
m \operatorname{ord}_{V} \eta=\sum_{W \in U} n(W) c(R, V, W)
$$

for all $V \in U$.

(0) Introductory note on (3.5). The proof of (3.5) is contained in its statement. In notes (I), (III), and (IV), we shall use (3.5) to exclude certain finite $U \subset D(R)^{\Delta}$ from being equal to $\mathfrak{D}(R, J)$ for any special $J$. In note (II) we shall use (3.5) to prove a partial converse of (3.5). For any $V \in D(R)^{\Delta}$ and $\theta \in L$ we put $V(\theta)=\operatorname{ord}_{V} \theta$.

(I) First note on (3.5). Let $R_{1} \in Q_{1}(R)$ and $R_{2} \in Q_{1}\left(R_{1}\right)$ be given by the equations $M\left(R_{1}\right)=\left(x_{1}, y_{1}\right) R_{1}$ with $\left(x_{1}, y_{1}\right)=(x / y, y)$ and $M\left(R_{2}\right)=\left(x_{2}, y_{2}\right) R_{2}$ with $\left(x_{2}, y_{2}\right)=\left(x_{1}, y_{1} / x_{1}\right)$. Let $U=\{A, B\}$ with $A=o(R)$ and $B=o\left(R_{2}\right)$. Clearly $\zeta_{R}(B)=\left(x^{2}, x y^{2}, y^{3}\right)$ with

$(\bullet)$

$$
(B(x), B(y), B(\zeta(B)), B(\zeta(A)), A(\zeta(B)))=(3,2,6,2,2) .
$$

In the notation of $(3.5)$, using only $(\bullet)$ we deduce a contradiction thus. Clearly $B(\eta)=2$ or 3 . If $B(\eta)=2$, then $m=n(A)+2 n(B)$ with $2 m=2 n(A)+6 n(B)$ and subtracting 2 times the first equation from the second we get the contradiction $0=2 n(B)$. If $B(\eta)=3$, then $m=n(A)+2 n(B)$ with $3 m=2 n(A)+6 n(B)$ and subtracting 3 times the first equation from the second we get the contradiction $-n(A)=0$. 
(II) Second note on (3.5). For $1<t \in \mathbb{N}_{+}$and $1 \leq j \leq t$ let $R_{j} \in Q_{j}(R)$ be given by $M\left(R_{j}\right)=\left(x_{j}, y_{j}\right) R_{j}$ with $\left(x_{j}, y_{j}\right)=\left(x / y^{j}, y\right)$. Let $U=\{A, B\}$ with $A=o(R)$ and $B=o\left(R_{t}\right)$. Clearly $\zeta_{R}(B)=\left(x, y^{t}\right) R$ and we have

\section{(•)}

$$
(B(x), B(y), B(\zeta(B)), B(\zeta(A)), A(\zeta(B)))=(t, 1, t, 1,1) .
$$

In the notation of (3.5), upon letting $B(\eta)=r$ we have $1 \leq r \leq t$. Moreover

$(\bullet \bullet)$

$$
m=n(A)+n(B) \quad \text { with } \quad r m=n(A)+t n(B) .
$$

Thus $t \neq 2$, reconfirming a piece of the easy part of (3.3). Moreover, assuming $t \geq 3$ and taking

$$
(\eta, B(\eta), m, n(A), n(B), a)=\left(x+y^{2}, 2, t-1, t-2,1, x y^{t-2}\right)
$$

it follows that $\mathfrak{D}(R, J)=U$ for a special $J$, confirming a converse of (3.5).

(III) Third note on (3.5). For $1<t<\tau \in \mathbb{N}_{+}$and $1 \leq j \leq \tau$ let $R_{j} \in Q_{j}(R)$ be given by $M\left(R_{j}\right)=\left(x_{j}, y_{j}\right) R$ with $\left(x_{j}, y_{j}\right)=\left(x / y^{j}, y\right)$. Let $U=\{A, B, C\}$ with $A=o(R)$ and $B=o\left(R_{t}\right)$ and $C=o\left(R_{\tau}\right)$. Clearly $\zeta_{R}(B)=\left(x, y^{t}\right) R$ with $\zeta_{R}(C)=\left(x, y^{\tau}\right) R$ and we have

$(\bullet)\left\{\begin{array}{l}(B(x), B(y), B(\zeta(B)), B(\zeta(A)), A(\zeta(B)), B(\zeta(C)))=(t, 1, t, 1,1, t) \\ \text { and }(C(x), C(y), C(\zeta(C)), C(\zeta(A)), A(\zeta(C)), C(\zeta(B)))=(\tau, 1, \tau, 1,1, t) .\end{array}\right.$

Letting the notation be as in (3.5), we clearly have $(B(\eta), C(\eta))=(r, r)$ or $(t, \rho)$ with $1 \leq r \leq t<\rho \leq \tau$. We also have

\section{$(\bullet \bullet)$}

$$
m=n(A)+n(B)+n(C) .
$$

In the first case, $r m=n(A)+t n(B)+t n(C)$ with $r m=n(A)+t n(B)+\tau n(C)$, and subtracting the first equation from the second equation we get the contradiction $0=(\tau-t) n(C)$. In the second case we have $t m=n(A)+\operatorname{tn}(B)+\operatorname{tn}(C)$ with $\rho m=n(A)+t n(B)+\tau n(C)$, and subtracting the first equation from $t$ times $(\bullet \bullet)$ we get the contradiction $0=(t-1) n(A)$. Thus there is no special $J$ with $\mathfrak{D}(R, J)=U$.

$(I V)$ Fourth note on (3.5). To sharpen the third note, in (3.5) assume $3 \leq|U|=$ $\tau+1$ and label the members of $U$ as $\left(A_{i}\right)_{0 \leq i \leq \tau}$. Put $A_{i}\left(\zeta_{R}\left(A_{j}\right)\right)=t_{i j}$. Assume $o(R)=A_{0}, t_{0 i}=1=t_{i 0}$ for $0 \leq i \leq \tau, 1<t_{11}=t_{1 j}=t_{21}<t_{22}$ for $1 \leq j \leq \tau$, $t_{22}=t_{2 j^{\prime}}$ for $2 \leq j^{\prime} \leq \tau$, and for each $\eta \in M(R) \backslash M(R)^{2}$ we have

either $(1) A_{1}(\eta)=A_{2}(\eta)$

or $(2) A_{1}(\eta)=t_{11}$. Now by (3.5) it follows that

(3) $m=\sum_{0 \leq i \leq \tau} n\left(A_{i}\right)$, and we have

either (4) $A_{1}(\bar{\eta}) m=n\left(A_{0}\right)+\sum_{1 \leq i \leq \tau} t_{11} n\left(A_{i}\right)$ with $A_{1}(\eta) m=n\left(A_{0}\right)+t_{11} n\left(A_{1}\right)+\sum_{2 \leq i \leq \tau} t_{22} n\left(A_{i}\right)$

or (5) $t_{11}(\eta) m=n\left(A_{0}\right)+\sum_{1 \leq i \leq \tau} t_{11} n\left(A_{i}\right)$.

If (4), then subtracting its first equation from its second equation we get the contradiction $0=\sum_{2 \leq i \leq \tau}\left(t_{22}-t_{11}\right) n\left(A_{i}\right)$. If (5), then subtracting it from $t_{11}$ times (3) we get the contradiction $0=\left(t_{11}-1\right) n\left(A_{0}\right)$. Thus there is no special $J$ with $\mathfrak{D}(R, J)=U$. To exhibit a specific $U$ with the above properties, take any integers $1<l(1)<\cdots<l(\tau)$. For $1 \leq j \leq l(\tau)$ let $R_{j} \in Q_{j}(R)$ be given by $M\left(R_{j}\right)=\left(x_{j}, y_{j}\right) R$ with $\left(x_{j}, y_{j}\right)=\left(x / y^{j}, y\right)$. Take $A_{i}=o\left(R_{l(i)}\right)$ for $1 \leq i \leq \tau$. 
Remark 3.6. Parts (I), (III), and (IV) of (3.5) provide examples of finite $U \subset D(R)^{\Delta}$ such that $o_{R}^{-1}(U)$ is globally unforked but there is no special pencil $J$ at $R$ for which $\mathfrak{D}(R, J)=U$, and so the converse of $(3.3)(\mathrm{I})$ does not hold.

Remark 3.7. As hinted in the Introduction, results about dicriticals or big stars, and so all the results of Propositions 3.1 to 3.5, can be paraphrased in terms of the language of complete ideals. For instance, the special primary ideal $J_{0}=$ $\left(a_{0}, b_{0}\right) R_{0}$ constructed in the situation of Proposition 3.4(V) is a reduction of the complete ideal $I=\prod_{0 \leq l \leq \mu} \widehat{J}_{i_{l}}^{p_{l}}$, where $\left(\widehat{J}_{i}\right)_{0 \leq i \leq \nu}$ is the simple $V$-ideal sequence of $R$ mentioned in $(2.4)(\mathrm{I})$ and $p_{0}, \ldots, p_{\mu}$ are suitable positive integers.

\section{REFERENCES}

[Ab1] S. S. Abhyankar, On the valuations centered in a local domain, American Journal of Mathematics, vol. 78 (1956), pages 321-348. MR0082477 (18:556b)

[Ab2] S. S. Abhyankar, Ramification Theoretic Methods in Algebraic Geometry, Princeton University Press, 1959. MR0105416 (21:4158)

[Ab3] S. S. Abhyankar, Resolution of Singularities of Embedded Algebraic Surfaces, First Edition of 1966 published by Academic Press; Second Enlarged Edition of 1998 published by Springer-Verlag. MR1617523 (99c:14021)

[Ab4] S. S. Abhyankar, Lectures on Algebra I, World Scientific, 2006. MR2253320 (2007k:13001)

[Ab5] S. S. Abhyankar, Inversion and Invariance of Characteristic Terms, Part I, Developments in Mathematics, Springer, 2010.

[Ab6] S. S. Abhyankar, Dicritical Divisors and Jacobian Problem, Indian Journal of Pure and Applied Mathematics, vol. 41 (2010), pages 77-97. MR2650101

[Ab7] S. S. Abhyankar, Pillars and Towers of Quadratic Transformations, Proceedings of the American Mathematical Society, to appear.

$[\mathrm{AbH}]$ S. S. Abhyankar and W. J. Heinzer, Existence of dicritical divisors, American Journal of Mathematics, to appear.

[AbL] S. S. Abhyankar and I. Luengo, Algebraic theory of dicritical divisors, American Journal of Mathematics, to appear.

[ZaS] O. Zariski and P. Samuel, Commutative Algebra, Volume II, Springer-Verlag, 1975. MR0389876 (52:10706)

Department of Mathematics, Purdue University, West Lafayette, Indiana 47907

E-mail address: ram@cs.purdue.edu 